\title{
Graphene-oxide coated LPGs for humidity sensing applications
}

Sahoo, Namita, Sun, Zhongyuan, Zhou, Kaiming, Chen, Xianfeng, Tan, Yidong, et al.

Namita Sahoo, Zhongyuan Sun, Kaiming Zhou, Xianfeng Chen, Yidong Tan, Lin Zhang, "Graphene-oxide coated LPGs for humidity sensing applications," Proc. SPIE 11355, Micro-Structured and Specialty Optical Fibres VI, 1135505 (1 April 2020); doi: 10.1117/12.2555365

SPIE. Event: SPIE Photonics Europe, 2020, Online Only 


\title{
Graphene-oxide coated LPGs for humidity sensing applications
}

\author{
Namita Sahoo ${ }^{\text {a, }}$, Zhongyuan Sun a , Kaiming Zhou a , Xianfeng Chen ${ }^{b}$, Yidong Tan ${ }^{c}$, Lin Zhang a \\ ${ }^{a}$ Aston Institute of Photonic Technologies, Aston University, Birmingham, United Kingdom \\ ${ }^{\mathrm{b}}$ School of Electronic Engineering, Bangor University, Bangor, United Kingdom \\ ${ }^{c}$ Department of Precision Instrument, Tsinghua University, Beijing, China \\ *E-mail: $\underline{\text { sahoon@aston.ac.uk }}$
}

\begin{abstract}
In this paper, we report a highly effective relative humidity (RH) sensor implemented on graphene oxide (GO) coated long period grating (LPG). The GO nanocolloides bonded onto a cylindrical fibre cladding enables the LPG with strong evanescent waves to absorb more water molecules increasing its RH sensitivity. In an LPG, the phase matching condition occurs when a forward propagating core mode is coupled with the co-propagating lower order cladding modes generating evanescent waves to interact with the surrounding medium. This unique effect of LPGs can be more enhanced with multilayer GO deposition. There is an expansion of GO film with the absorption of more water molecules as RH increases. The absorption of water molecules on GO coating increases the conducting carrier (holes) density on it, thus decreasing the refractive index of GO film. The combined effect of increasing evanescent waves and modulated refractive index makes the GO coated LPGs as effective RH sensors. Our recently achieved results have shown the RH sensitivity of the GO coated LPG is about $0.01 \mathrm{~dB} / \% \mathrm{RH}$. We have also investigated the effect on GO layer thickness, showing thicker layer increases the RH response of the LPG cladding mode resonances in lower wavelength region.
\end{abstract}

Keywords: Long period fibre gratings, Graphene oxide deposition, Humidity sensing

\section{Introduction}

Relative humidity $(\mathrm{RH})$ sensing creates a major role in food processing industry, structural health monitoring and many other environmental sensing applications [1] [2]. Besides, comparing with resistor and capacitive type of sensors, the optical fibre sensors are more advantageous as they are highly sensitive, structurally very simple, immune to electromagnetic interference, resistant to corrosive environment etc. In recent works, many fibre sensors, such as fibre Bragg gratings (FBGs) made in polymer fibre [3] and long period gratings (LPGs) with different refractive index coatings [4], have been explored for different RH sensing applications.

Long Period gratings have typical periods ranging from tens to few hundred microns. The relatively large period structure enables the light coupling from the core mode to the low order cladding modes under phase matching condition, generating a set of attenuation bands centred at different wavelengths in transmission. The spectral distribution of each cladding mode depends on the fibre structure and surrounding medium. Thus, the wavelength shift of the LPG attenuation bands can be obtained with the change in refractive index of surrounding medium as the effective refractive index of the core and cladding modes varies [5]. This is the mechanism of an LPG acting as a refractive index sensor.

Graphene is a planar sheet of carbon material which consists of $\mathrm{sp}^{2}$ bonding structure. It has excellent electronic and optical property that has been explored in fibre sensors for various applications such as RH sensing for environmental monitoring and bioscience applications. Graphene has advantages of large surface area, mechanical stability and good thermal and electrical conductivities [6]. Particularly, the tuneable optical properties of graphene makes it more suitable for fibre grating sensors. It has been reported that the graphene oxide (GO) adsorbs water molecules that enhances the conducting carrier density, thus decreases the effective refractive index of GO film with increase in RH.

In this paper, we present a multilayer GO-coated LPG as an RH sensor device. A dip coating technique was used to deposit GO on to the LPG surface. As GO absorbs water molecules, the effective refractive index of cladding modes changes, enabling the humidity change to be monitored. The refractive index of GO film has real and imaginary part, and in RH sensing, the imaginary part of the GO index is significantly affected by the water absorption. Our proposed sensor can be extended to more advanced work with fabricating biosensors by varying the GO-coating thickness. 


\section{Theoretical background}

For an LPG, the light coupling occurs between a core mode and a co-propagating cladding mode as shown in Fig. 1. The resonant wavelength $\lambda_{\text {res }}$ is defined as:

$$
\lambda_{\text {res }}=\left(n_{c o}^{e f f}-n_{c l, m}^{e f f}\right) . \Lambda
$$

where $n_{c o}^{e f f}$ and $n_{c l, m}^{e f f}$ are the effective refractive indices of the core mode and the m-th cladding mode respectively and $\Lambda$ is the grating period. As the factor $\left(n_{c o}^{e f f}-n_{c l, m}^{e f f}\right) \ll 1$, the period of a forward coupled LPG is much larger than that of the backward-coupled FBG.

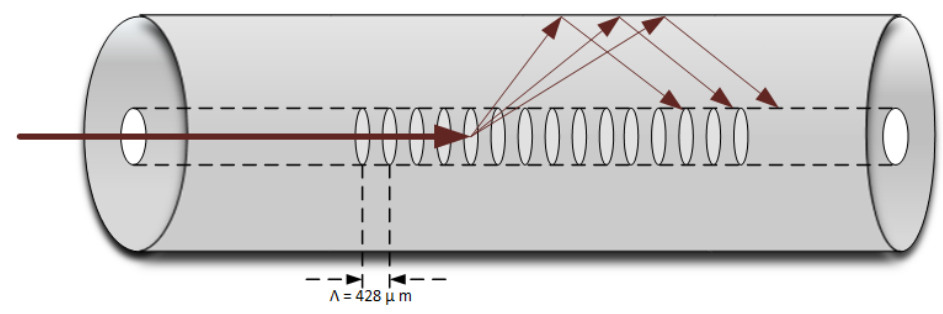

Figure1. Schematic of mode-coupling of long period grating (LPG).

The cladding mode $(\mathrm{m}=1,2,3,4, \ldots)$ is a function of cladding refractive index and the surrounding medium index. Therefore, the wavelength shift of coupled cladding modes resonance can be obtained with the change in refractive index of surrounding medium as because $n_{c l, m}^{e f f}$ is affected.

The transmission of an LPG for the coupled cladding mode resonance is given by,

$$
T_{i}=1-\sin ^{2}\left(k_{i} L\right)
$$

From equation (2) it is clear that the length $(L)$ of the LPG and the coupling coefficient $\left(k_{i}\right)$ of the $i$-th cladding mode are the important parameters affecting the strength of resonance. When the LPG surface is coated with GO film, the cladding mode coupling coefficient will change with the refractive index of GO coating layer when it absorbs water, introducing a mechanism for RH sensing.

\section{Fabrication of the sensor}

The LPGs are fabricated using the point-by-point writing technique, where the periodic refractive index modulation of the fibre is realised by a collimated UV beam exposure. A frequency-doubled Argon-ion laser (Innova 300C MotoFred) generating $244 \mathrm{~nm}$ UV emission is focused via a lens system onto the normal B/Ge photosensitive fibre core. The motion driver (Newport, Model XPS) is used to control the shutter speed with 50:50 duty cycle through a software controlled programme. The inscription parameters such as writing speed and grating length are specified at the beginning of fabrication. The LPGs were designed with $428 \mu \mathrm{m}$ period and a length of $20 \mathrm{~mm}$.

The GO nanocolloids solution with concentration of $2 \mathrm{mg} / \mathrm{mL}$ was bought from Sigma-Aldrich. In the GO deposition process, firstly, the LPG surface was cleaned with methanol and then deionized (DI) water multiple times. Then the fibre surface was silanized by treating it in 1molar sodium hydroxide $(\mathrm{NaOH})$ solution that will enrich a number of hydroxyl $\left(\mathrm{OH}^{-}\right)$group onto it to bind the GO film more effectively. The LPG was then immersed into a 5\% of (3-Aminopropyl) triethoxysilane (APTES) solution to form Si- O- Si bonding onto the grating surface. Finally, we made a GO solution with DI water with $1.2 \mathrm{mg} / \mathrm{mL}$ of GO concentration and we used this solution for multilayer deposition for the LPGs. After 
each coating, the fibre was dried by placing it onto a hotplate with set temperature at $55^{\circ} \mathrm{C}$ for approximately 40 minutes, and then the second coating was applied following the same procedure.

Figure 2 is the image of a GO-coated LPG using an optical microscope (Carl Zeiss) by an objective of 20X. This clearly shows the GO coated layer is relatively uniform across the LPG surface. The spectrum of the LPG was measured after the GO coating and plotted with the original spectrum as shown in Fig. 3. It is clear from the figure that the attenuation bands of the three coupled cladding modes have significantly reduced after the GO coating, especially for the peak at $1650 \mathrm{~nm}$, the strength reduced from $15 \mathrm{~dB}$ to only $4 \mathrm{~dB}$. This is due to the change in cladding mode coupling coefficient induced by the extra layer of GO film on the LPG surface.

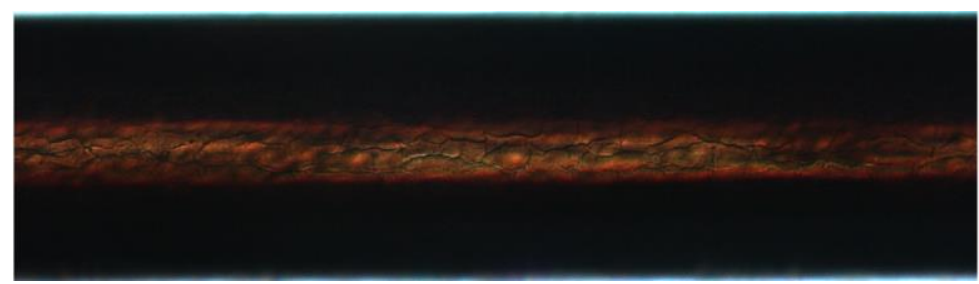

Figure 2. The microscope image of the GO- coated LPG.

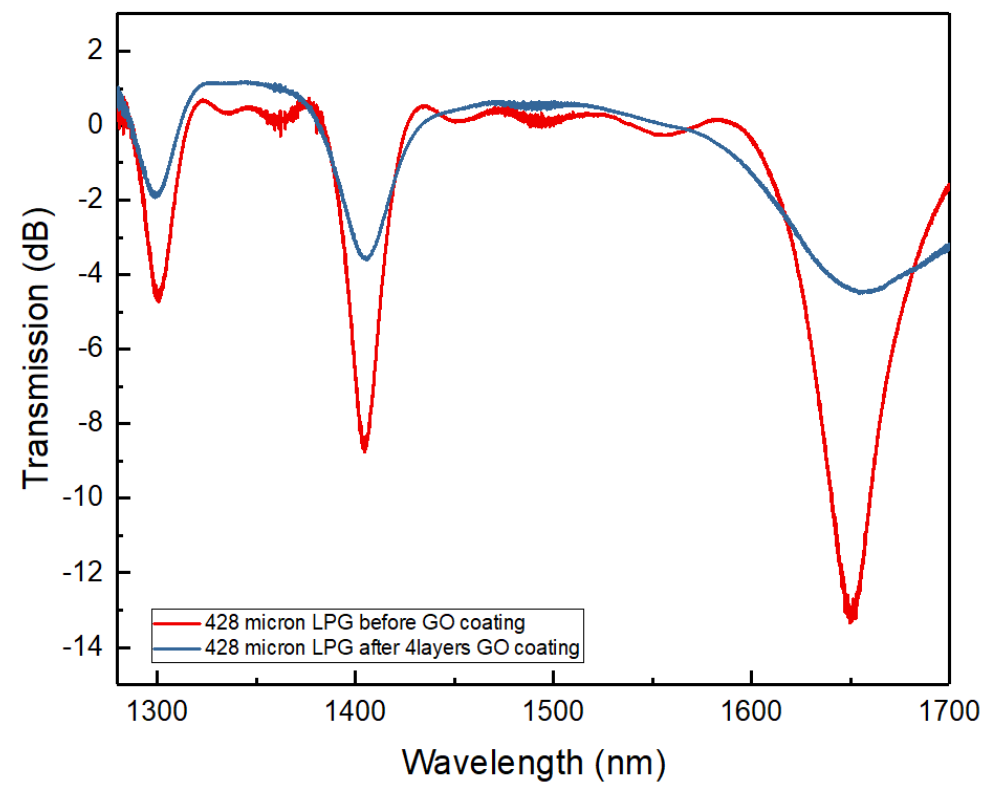

Figure 3. LPG spectra before and after GO coating.

\section{Experimental setup}

Figure 4 shows the experimental setup for RH sensing measurement. We used a humidity chamber (Binder KBF 115) and the GO-coated LPG was placed inside the chamber. The two fibre ends were taken out from the chamber and one end was connected with the broadband light source and the other end was connected to the optical spectrum analyser. Initially, the chember was set at a constant temperature at $25^{\circ} \mathrm{C}$ and with an RH of $20 \%$ for aprroximately 30 mins to get a stabilised RH environment inside the chamber. For the actual measurement, the RH was varied from $40 \%$ to $80 \%$ with a step of $10 \%$. 
When changing the RH, a 10mins setting time was maintained to reach the constant value of the RH and then the LPG spectrum was recorded.

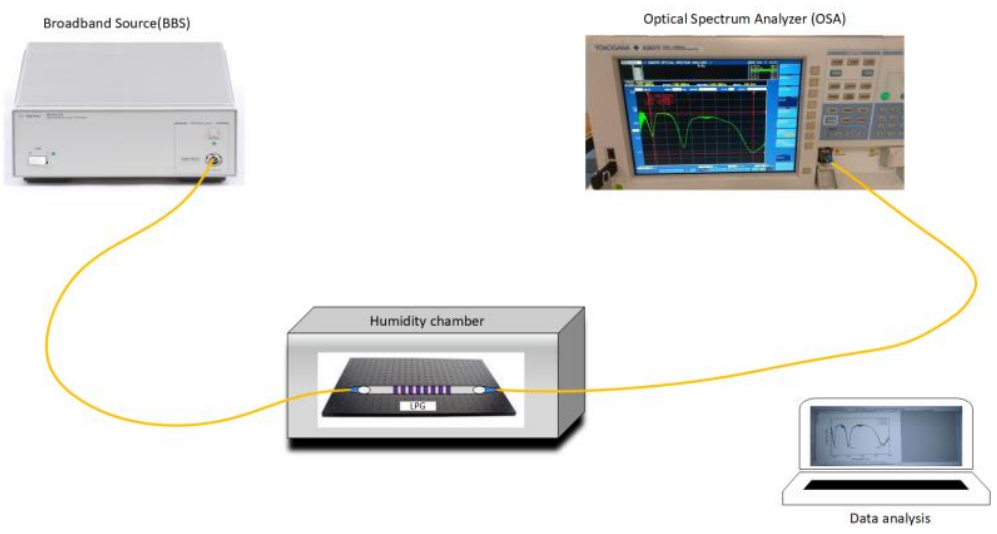

Figure 4. Experimental Setup for humidity sensing measurement

As to examine the effect of GO film thickness on LPG for the RH response, initially we measured the LPG with 2-layer of GO coating After the first set of measurement, the LPG was further coated with two more GO layers following the same procedure. When the grating was totally dried, the 4-layer GO coated LPG was subjected to the RH measurement and the results were recorded and compared with that of 2-layer GO-coated grating.

\section{Results and discussion}

Fig. 5 and 6 show the RH sensitivities of the 2-layer and 4-layer GO-coated LPGs. From Fig. 5a, we clearly see that only Peak 3 at $\sim 1650 \mathrm{~nm}$ of the 2-layer GO-coated LPG responded to RH change. However, after the deposition of two more layers, all three peaks of the 4-layer GO-coated LPG have responded to the RH change, as shown in Fig. 6a. In some previousely reported work, the GO-coated gratings (LPG and TFG) have shown both amplitude variation and wavelength shift under RH change [7] [8]. In our GO-coated LPGs, the resonance amplitude change is more pronounced whereas the shift of resonance peak hardly visible. This may be explained that the GO coating on our LPGs may have limited thickness, thus only the absorption, which is associated with the imaginary part of the GO refractive index, is dominant.
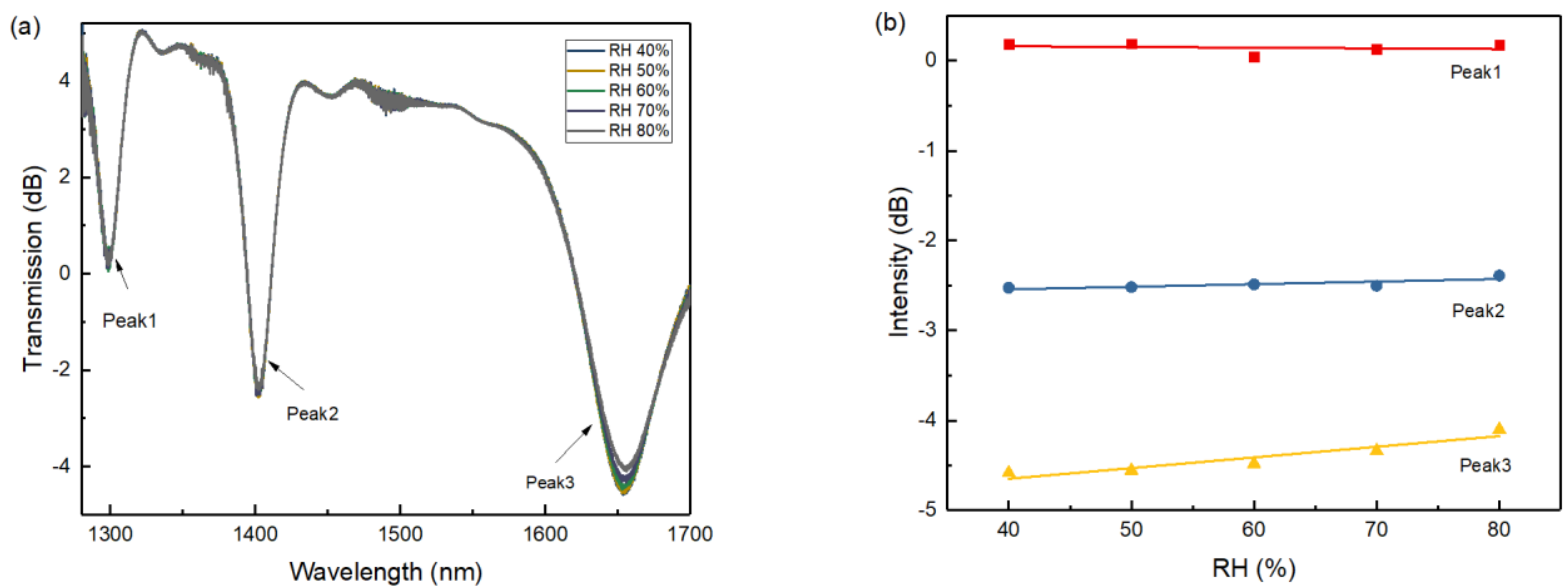

Figure 5. (a) Transmission spectra of 2-layer GO-coated LPG for different RH changes; (b) Peak value vs RH (\%) plot for three resonance peaks. 

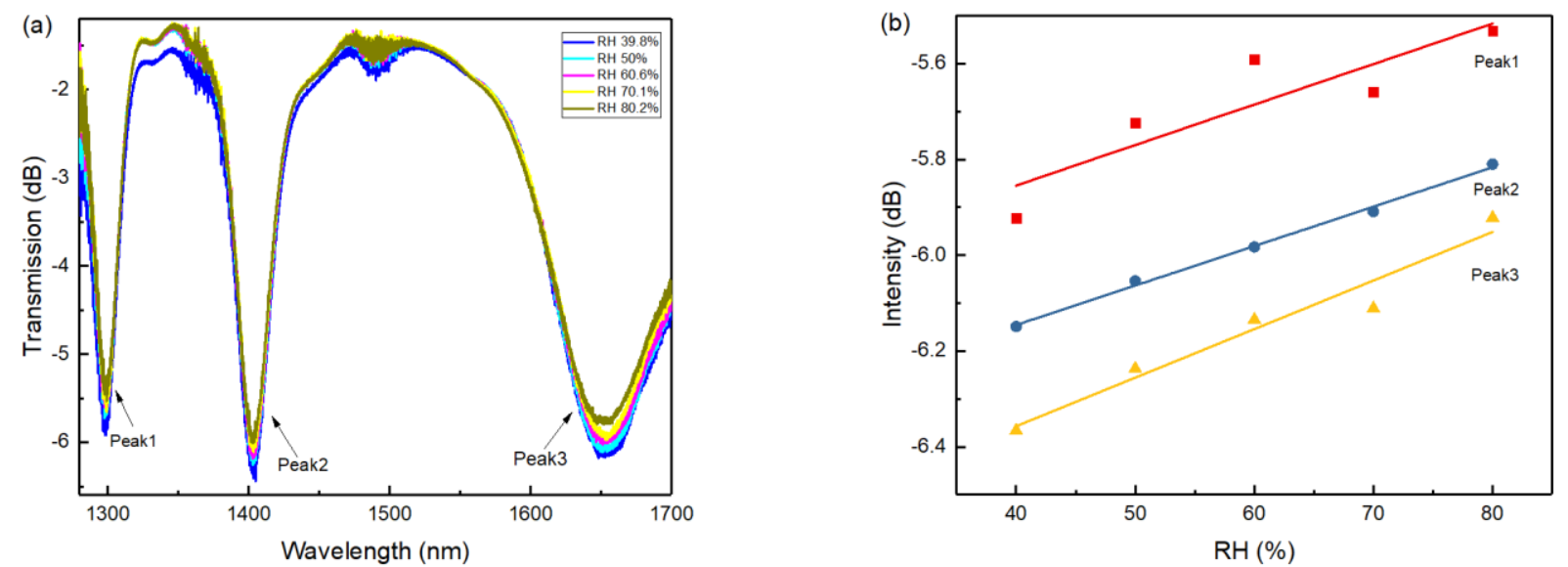

Figure 6. (a) Transmission spectra of 4-layer GO-coated LPG for different RH changes; (b) Peak value vs RH (\%) plot for three resonance peaks.

We have plotted the attenuation peak amplitude against RH change for the 2-layer and 4-layer GO-coated LPGs in Figure $5 \mathrm{~b}$ and $6 \mathrm{~b}$, from which we can estimate the RH sensitivities for the two gratings. It is noted from the figures that the $\mathrm{RH}$ sensitivity of the longest wavelength peak (Peak 3 at $\sim 1650 \mathrm{~nm}$ ) is the highest at $\sim 0.01 \mathrm{~dB} / \% \mathrm{RH}$, but it did not change very much after two more layers coating. However, for Peak 1 at $\sim 1300 \mathrm{~nm}$, the RH sensitivity increased from $0.00025 / \% \mathrm{RH}$ for the 2-layer coating to $0.0084 / \% \mathrm{RH}$ for the 4-layer coating, enhanced by 33 times. While for Peak 2 at $\sim 1400 \mathrm{~nm}$, the $\mathrm{RH}$ sensitivity increased from $0.0028 / \% \mathrm{RH}$ for the former to $0.0082 / \% \mathrm{RH}$ for the latter, enhanced by $\sim 4$ times. Due to limited facility, we were not able to measure the actual thickness of the GO-coating. We believe with more controllable and measurable GO-coating, the RH response of the LPGs can be improved.

Finally, in order to evaluate how RH level was maintained in the environment chamber, we investigated the stability of RH measurement by using the 4-layer GO-coated LPG. In the experiment, the RH level was varied from $30 \%$ to $80 \%$ with an interval of $10 \%$. We set two minutes for the environment chamber to reach the new level, and took five measurements by recording the spectrum of the grating with an interval of 2 minutes. Fig. 7 plots the humidity response with time, and it clearly shows that the GO-coated LPG responds to RH change very rapidly and maintains constant while the RH level is unchanged.

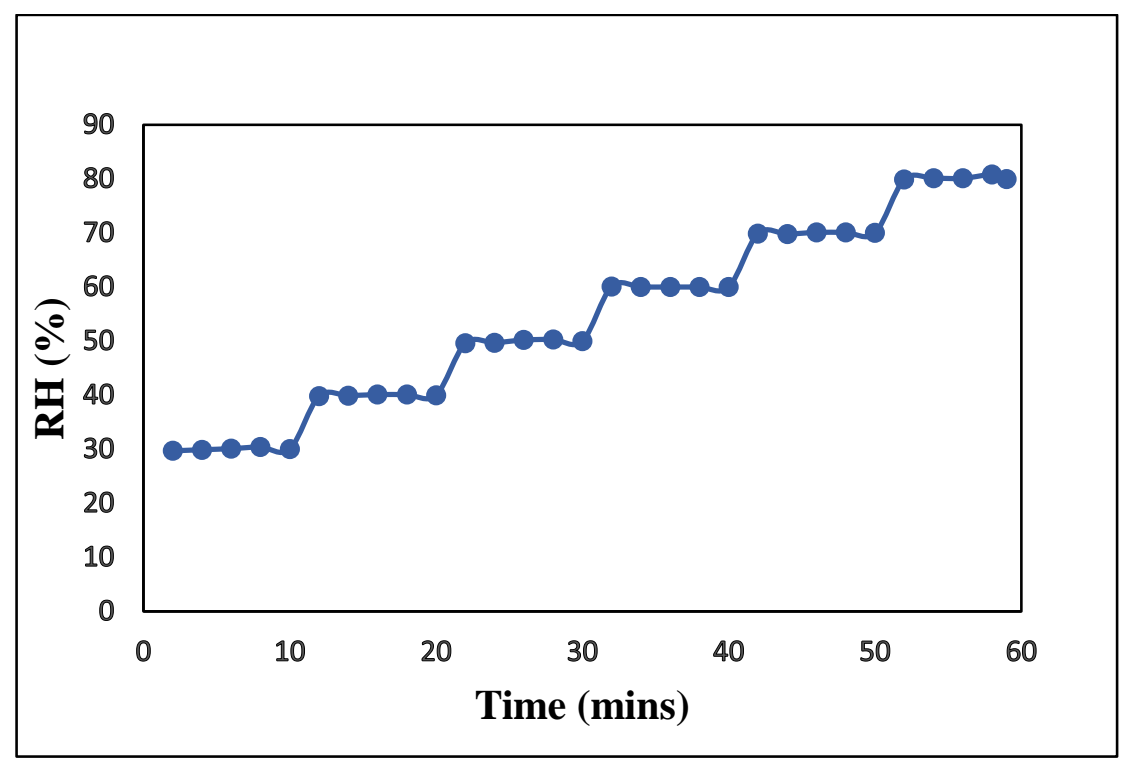

Figure 7. Multiple measurements of RH response of the 4-layer GO-coated LPG. 


\section{Conclusion}

An RH sensor based on LPG with GO coating has been implemented. The GO was deposited onto the grating surface after the LPG was silanized with APTES solution for enhanced bonding of the GO-nanosheets. We have evaluated the RH response of the LPGs with 2-layer and 4-layer GO-coating and the results have clearly showed that thicker GO coating may increase the RH sensitivity. The GO-coated LPG has also showed good constant response to certain RH values. These convincing results may open the opportunity for high RH sensitivity sensors by fabricating LPGs in mid IR range with thicker GO layer deposition, and these sensors may be deployable for gas and environment sensing applications.

\section{Acknowledgment}

This project is supported by the UK Royal Society Newton Advanced Fellowship programme (NAF/R1/191072) and European Union Horizon 2020 Research and Innovation Staff Exchange (RISE) programme (No. 872049, IPN-Bio).

\section{References}

1. Enjin, A., et al., Humidity Sensing in 〈em>Drosophila</em>. Current Biology, 2016. 26(10): p. $1352-1358$.

2. Hill, K.O., et al., PHOTOSENSITIVITY IN OPTICAL FIBER WAVEGUIDES - APPLICATION TO REFLECTION FILTER FABRICATION. Applied Physics Letters, 1978. 32(10): p. 647-649.

3. Webb, D.J., Fibre Bragg grating sensors in polymer optical fibres. MEASUREMENT SCIENCE and TECHNOLOGY, 2015. 26(9).

4. Esposito, F., et al., Ultrasensitive biosensor based on long period grating coated with polycarbonate-graphene oxide multilayer. Sensors and Actuators B: Chemical, 2018. 274: p. 517-526.

5. Patrick, H.J., A.D. Kersey, and F. Bucholtz, Analysis of the response of long period fiber gratings to external index of refraction. Journal of Lightwave Technology, 1998. 16(9): p. 1606-1612.

6. Chen, D., H. Feng, and J. Li, Graphene Oxide: Preparation, Functionalization, and Electrochemical Applications. Chemical Reviews, 2012. 112(11): p. 6027-6053.

7. Bing Xu, Jun Huang, Liyun Ding and Jun Cai, Graphene oxide-functionalized long period fiber grating for ultrafast label free glucose biosensor. Materials Science \& Engineering C 107 (2020) 110329.

8. Jiang, B., et al., Graphene-induced unique polarization tuning properties of excessively tilted fiber grating. Optics Letters, 2016, Vol. 41, No. 23: p. 5450. 